International Journal of Agricultural Policy and Research Vol.10 (1), pp. 16-25, January 2022

Available online at https://www.journalissues.org/IJAPR/

https://doi.org/10.15739/IJAPR.22.003

Copyright (C) 2022 Author(s) retain the copyright of this article

ISSN 2350-1561

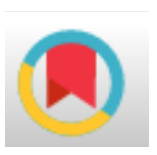

Original Research Article

\title{
Comparative effect of fertilization on cocoyam and component crops on Cocoyam-based intercropping systems in Uyo, Southeastern Nigeria
}

* Udounang, P.I., Ekwere, 0. J. and

Akpainyang, F. E.

Department of Crop Science, Akwa Ibom State University, Obio Akpa Campus.

*Corresponding Author Email: patunang@yahoo.com

Tel.: +2348028455194
Field experiment was conducted at University of Uyo Teaching, research farm during the 2018 and 2019 cropping seasons to assess the effect of fertilization on cocoyam-based intercropping systems. The experiment was laid out in a randomized complete block design with three replications.

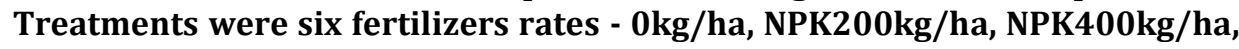
PM2.5t/ha, PM5.0t/ha, Mixture (NPK200kg/ha + PM2.5t/ha) and six crop mixtures - Sole Cocoyam, Sole Melon, Sole Maize, Cocoyam + Maize, Cocoyam + Melon and Cocoyam + Melon + Maize). Result analysed with analysis of variance revealed PM2.5t/ha, NPK400kg/ha and Mixture (NPK200kg/ha + PM2.5t/ha) gave higher plant height, leaf area, stem girth in sole cocoyam, maize and melon respectively. PM2.5t/ha favoured leaf area, stem girths of cocoyam, maize with cocoyam + maize, cocoyam + melon and cocoyam + melon + maize crop mixtures. PM5.0t/ha gave higher values of corm, cormels, cormels weight, cormels number and cormels length in both sole cocoyam and mixtures (cocoyam + melon, cocoyam + maize, cocoyam + melon + maize) in both seasons respectively. PM2.5t/ha gave higher values of maize grain yield with cocoyam + maize, cocoyam + melon + maize crop mixtures. PM5.0t/ha and NPK400kg/ha gave higher melon seed values with cocoyam + melon and cocoyam + Melon + maize. Conclusively, it is recommended that application of organic fertilizer promotes higher yields and yield components in both sole and crop mixtures of cocoyam and maize respectively.

Keywords: Poultry manure (PM), NPK 15:15:15, Cocoyam, melon, maize, corms, cormels

\section{INTRODUCTION}

Marginal lands not normally productive, have been converted to fertile farmlands because of effective soil fertility management through use of manures, inorganic fertilizers and soil amendments. Fertilizers are generally known to increase yields per hectare of crops in a planting regime (Udounang, 2007). It could therefore be said that fertilizers are essentially chemical nutrients (organic and inorganic) desired by crops at different levels and time for good growth and yield. The nutrient elements in fertilizers are important in boosting crop production(Udounang
,2007).

Crop production in general and crop growth, yield in particular has been influenced by soil degradation, soil fertility status and crop production depends to a large extent on the judicious use of the resources. In tropical regions, soil fertility declines due to soil degradation and most tropical crops require certain amount of nutrients for growth and productivity (Wong et al., 2003). Tropical soils are adversely affected by sub-optimal soil fertility and erosion, deterioration of nutrients status and changes in 
soil organisms (Economic Commission for Africa, 2011). Continuous declines in soil fertility in Nigeria have prevented farmers from obtaining yields in crops. The use of inorganic fertilizers improves the crop yields by way of increasing soil fertility and nutrient availability (Smil, 2000). NPK fertilizer can stimulate crop growth and improve its mineral uptake (Azza et al., 2006).

Intercropping on the other hand involves the growing of two or more crops simultaneously in the same season on the same piece of land. It is a common production practice among the developing countries such as Nigeria (Ofori and Stern, 1987). Intercropping strategies involves the planting of deep rooted crops with shallow rooted crops or planting tall crops with shorter crops that require partial shade. Most common goal is the production for greater yield on a given piece of land using resources or ecological processes that would otherwise not be utilized by single crop (Oluma, 2010). The system involves numerous associated crops such as cocoyam, maize, cowpeas, plantains and vegetables depending on the agro ecological zone (Doku, 1988).

Cocoyam which is a monocotyledonous crop, belongs to the family - Araceae and is one of the most staple food in Africa. In Nigeria, it is assumed to take third position after yam and cassava (Knipsheer and Wilson, 1980). Cocoyam is grown basically for the corms and cormels, though the leaves, petiole are often times eaten as vegetables in some tribes and countries. Cocoyam grown as sole has been confirmed to perform well with both organic and inorganic fertilizers giving best growth and yield with NPK $200 \mathrm{~kg} / \mathrm{ha}$ and with organic manure (poultry manure) at 2.5 tonnes/ha (Udounang, 2007). Similar works were done on tillage practices (Udounang, 2015) with the affirmation that cocoyam does well with mounds in comparison with ridges and flat. However, the potential performance of cocoyam in an intercropping systems especially with crops of relative importance were yet to be explored. The aim of the research therefore is to study performance of cocoyam in an intercropping system using different rates of both organic and inorganic fertilizers.

\section{MATERIALS AND METHODS}

The field experiment was carried out at the University of Uyo Teaching and research farm. The site is located at latitude $5^{\circ} 17$ and $5^{\circ} 27 \mathrm{~N}$, longitude $7^{\circ} 27$ and $7^{\circ} 58 \mathrm{E}$ with an altitude of $38.1 \mathrm{~m}$ above sea level. It has about $2500 \mathrm{~mm}$ rainfall annually. The rainfall pattern is bimodal with long (March - July) and short (September - November) rainy seasons separated by a short dry spell of uncertain length usually during the month of August. The mean relative humidity is $30^{\circ} \mathrm{C}$. The mean sunshine hours are 12 (Peters et al., 1989). The experiment was laid out in a Randomized Complete Block Design with three replications. The treatments were six (6) rates of fertilizers $(0 \mathrm{~kg} / \mathrm{ha}$, NPK200kg/ha, NPK400kg /ha, Poultry Manure2.5t/ha, Poultry Manure 5.0t/ha, Mixture- NPK200kg/ha + Poultry Manure 2.5t/ha) and six (6) crop mixtures (Sole Cocoyam,
Sole Melon, Sole Maize, Cocoyam + Maize, Cocoyam + Melon and Cocoyam + Melon + Maize). The plot sizes were $23 \times 6$ $\mathrm{m}$ and $3 \times 6 \mathrm{~m}$ respectively.

The cocoyam setts with average weight of $0.3 \mathrm{~kg}$ were planted on the crest of the mounds at $1 \times 1 \mathrm{~m}$ spacing to give a plant population of 10,000 plants/hectare. The maize seeds were planted at 3 seeds per stand on the side of the mound at $1 \times 0.5 \mathrm{~m}$ spacing and later thinned to 2 plants per stand. Three melon seeds were planted per stand and later thinned to 2 plants per stand on the crest of the mound at $1 \times 1 \mathrm{~m}$ spacing. The poultry manure was sun cured for about 3days and de-caked before application. Both the NPK and the Poultry Manure were applied in band at 2 months after planting (MAP). Weeding was done manually at 4 months' interval after planting while harvesting was done at 4 months after planting for maize, melon and 8 months after planting (MAP) for cocoyam.

The following growth and yield parameters were collected - plant height, leaf area, stem girth for growth and corms yield, cormels yield and number of cormels per plant for yield parameters.

The data obtained were subjected to analysis of Variance and treatment means were separated using F- LSD at 5\% probability level.

\section{RESULT}

Results showed significant difference $(\mathrm{P}<0.05)$ among the intercrop and fertilizers levels on the cocoyam height (Table 1). With sole cocoyam and cocoyam + melon crop mixture, the fertilizer level of NPK200kg/ha gave a higher value of cocoyam height at 2, 4, 6 Months After Planting (MAP) in both planting seasons. Conversely, lower values of cocoyam height were recorded with the mixture (NPK200+ Pm 2.5) with sole and cocoyam + melon intercrop at 2, 4, 6 MAP in both seasons. With intercrop of cocoyam + maize and cocoyam + maize + melon, Poultry Manure (Pm)2.5t/ha gave a higher value of cocoyam height whereas NPK400kg/ha gave the least value at 2, 4, 6 MAP in both planting seasons.

The effect of intercrop and fertilizer levels on the leaf area of cocoyam is shown in Table 2. Result showed significant difference $(\mathrm{P}<0.05)$ at 2,6 MAP in fertilizer levels and $6 \mathrm{MAP}$ in intercrop in both planting seasons. At $2 \mathrm{MAP}$, NPK $200 \mathrm{~kg} / \mathrm{ha}$ gave a higher value of the leaf area whereas Pm2.5t/ha recorded the highest value of leaf area at 4, 6 MAP with sole cocoyam. For intercrop of cocoyam + melon, cocoyam + maize and cocoyam + maize + melon, Pm2.5t/ha gave higher values of leaf area at 2, 4, 6 MAP in both seasons.

Table 3 shows the effect of intercrop and fertilizer levels on the stem girth of cocoyam. Results showed significant difference $(\mathrm{P}<0.05)$ on intercrop at 2,4 MAP and fertilizer levels at 2, 4, 6 MAP in both planting seasons. For sole cocoyam and intercrop of cocoyam + melon, cocoyam + maize, Pm2.5t/ha gave the highest value of stem girth of cocoyam at 2, 4, 6 MAP in the two years of planting whereas 
Table 1. Effect of intercrop and fertilizer levels on the height of Cocoyam

\begin{tabular}{|c|c|c|c|c|c|c|c|}
\hline \multirow{3}{*}{$\begin{array}{l}\text { Treatments } \\
\text { Crop mixtures }\end{array}$} & \multirow{3}{*}{ Fertilizer levels } & \multicolumn{6}{|c|}{ Months After Planting (cm)(MAP) } \\
\hline & & \multicolumn{2}{|c|}{2} & \multicolumn{2}{|c|}{4} & \multicolumn{2}{|c|}{6} \\
\hline & & 2018 & 2019 & 2018 & 2019 & 2018 & 2019 \\
\hline \multirow[t]{6}{*}{ Sole cocoyam } & 0kg/ha & 51.2 & 49.5 & 75.7 & 74.2 & 91.9 & 92.0 \\
\hline & NPK200kg/ha & 60.23 & 58.2 & 90.6 & 92.1 & 121.5 & 122.6 \\
\hline & NPK400kg/ha & 59.27 & 56.3 & 90.4 & 91.4 & 120.1 & 120.8 \\
\hline & PM 2.5t/ha & 55.13 & 55.9 & 85.4 & 86.1 & 113.4 & 140.2 \\
\hline & PM 5.0t/ha & 54.87 & 53.5 & 75.2 & 74.8 & 93.9 & 95.2 \\
\hline & NPK200 + PM 2.5 & 47.33 & 48.4 & 73.7 & 72.5 & 91.7 & 92.4 \\
\hline \multirow[t]{6}{*}{$\mathrm{Co}+\mathrm{Me}$} & $0 \mathrm{~kg} / \mathrm{ha}$ & 50.8 & 48.2 & 76.2 & 76.0 & 101.6 & 110.0 \\
\hline & NPK200kg/ha & 61.0 & 62.0 & 91.2 & 93.1 & 130.6 & 131.2 \\
\hline & NPK400kg/ha & 63.9 & 64.2 & 90.1 & 91.2 & 122.0 & 128.5 \\
\hline & PM 2.5t/ha & 54.2 & 54.6 & 81.3 & 80.4 & 109.4 & 109.8 \\
\hline & PM 5.0t/ha & 50.7 & 51.0 & 76.0 & 75.2 & 101.0 & 107.4 \\
\hline & NPK200 +PM 2.5 & 51.3 & 52.1 & 75.4 & 74.8 & 100.0 & 105.0 \\
\hline \multirow[t]{6}{*}{$\mathrm{Co}+\mathrm{Ma}$} & $0 \mathrm{~kg} / \mathrm{ha}$ & 48.4 & 48.7 & 69.9 & 68.5 & 93.8 & 94.1 \\
\hline & NPK200kg/ha & 51.4 & 52.1 & 77.6 & 78.2 & 102.3 & 104.3 \\
\hline & NPK400kg/ha & 50.6 & 50.2 & 76.0 & 76.4 & 101.2 & 102.5 \\
\hline & PM 2.5t/ha & 64.0 & 63.8 & 96.3 & 94.4 & 128.0 & 127.8 \\
\hline & PM 5.0t/ha & 55.4 & 56.2 & 84.6 & 85.2 & 112.7 & 111.8 \\
\hline & NPK200 +PM 2.5 & 55.3 & 55.8 & 83.7 & 83.9 & 111.0 & 108.7 \\
\hline \multirow[t]{6}{*}{$\mathrm{Co}+\mathrm{Ma}+\mathrm{Me}$} & $0 \mathrm{~kg} / \mathrm{ha}$ & 50.4 & 49.2 & 75.0 & 74.5 & 98.7 & 99.1 \\
\hline & NPK200kg/ha & 51.9 & 50.8 & 77.7 & 78.0 & 103.8 & 101.6 \\
\hline & NPK $400 \mathrm{~kg} / \mathrm{ha}$ & 40.3 & 41.3 & 80.4 & 80.6 & 81.6 & 81.3 \\
\hline & PM 2.5t/ha & 54.9 & 55.0 & 82.9 & 83.2 & 111.5 & 107.4 \\
\hline & PM 5.0t/ha & 58.0 & 58.2 & 87.7 & 89.1 & 117.4 & 116.8 \\
\hline & NPK200 +PM 2.5 & 46.5 & 47.0 & 69.3 & 68.5 & 93.9 & 94.1 \\
\hline LSD (0.05)Crop mixture & & 130.3 & 131.2 & 356.7 & 362.3 & 533.2 & 524.3 \\
\hline LSD(0.05)Fertilizer levels & & 103.2 & 101.6 & NS & NS & 183.8 & 184.1 \\
\hline
\end{tabular}

$\mathrm{Co}=$ Cocoyam; $\mathrm{Me}=$ Melon; $\mathrm{Ma}=$ Maize; $\mathrm{NPK}=$ Nitrogen Phosphorus Potassium; $\mathrm{PM}=$ Poultry Manure

Table 2. Effect of intercrop and fertilizer levels on the leaf area of Cocoyam

\begin{tabular}{|c|c|c|c|c|c|c|c|}
\hline \multirow{3}{*}{$\begin{array}{l}\text { Treatments } \\
\text { Crop mixtures }\end{array}$} & \multirow[b]{3}{*}{ Fertilizer levels } & \multicolumn{6}{|c|}{ Months After Planting (cm²)(MAP) } \\
\hline & & \multicolumn{2}{|c|}{2} & \multicolumn{2}{|c|}{4} & \multicolumn{2}{|c|}{6} \\
\hline & & 2018 & 2019 & 2018 & 2019 & 2018 & 2019 \\
\hline \multirow[t]{6}{*}{ Sole cocoyam } & 0kg/ha & 770.6 & 780.1 & 1633.6 & 1634.6 & 2450.4 & 2449.6 \\
\hline & NPK200kg/ha & 937.6 & 930.2 & 1965.3 & 1966.2 & 2948.0 & 2955.0 \\
\hline & NPK400kg/ha & 913.0 & 901.4 & 1659.6 & 1661.2 & 2489.4 & 2451.2 \\
\hline & PM 2.5t/ha & 889.3 & 878.9 & 2008.4 & 2004.8 & 3012.6 & 3025.1 \\
\hline & PM 5.0t/ha & 833.8 & 840.5 & 1773.4 & 1770.4 & 2660.1 & 2589.2 \\
\hline & NPK200 +PM 2.5 & 901.2 & 890.4 & 1920 & 1921.1 & 2880.4 & 2780.4 \\
\hline \multirow[t]{6}{*}{$\mathrm{Co}+\mathrm{Me}$} & 0kg/ha & 731.8 & 730.4 & 1515.8 & 1510.5 & 2273.7 & 2272.4 \\
\hline & NPK200kg/ha & 1052.3 & 1055.6 & 1924.3 & 1925.0 & 2886.4 & 2877.5 \\
\hline & NPK400kg/ha & 848.7 & 840.5 & 1704.8 & 1710.2 & 2557.3 & 2577.3 \\
\hline & PM 2.5t/ha & 1144.7 & 1201.0 & 1970.2 & 1989.4 & 2955.3 & 3001.4 \\
\hline & PM 5.0t/ha & 976.5 & 984.2 & 1615.9 & 1605.2 & 2423.0 & 2413.6 \\
\hline & NPK200 +PM 2.5 & 1097.6 & 1101.4 & 1891.1 & 1884.2 & 2836.6 & 2786.4 \\
\hline \multirow[t]{6}{*}{$\mathrm{Co}+\mathrm{Ma}$} & $0 \mathrm{~kg} / \mathrm{ha}$ & 657.9 & 661.2 & 1300.8 & 1301.1 & 1951.2 & 1960.1 \\
\hline & NPK200kg/ha & 942.7 & 940.8 & 1905.7 & 1890.4 & 2858.6 & 2744.2 \\
\hline & NPK400kg/ha & 817.3 & 811.6 & 1706.2 & 1699.5 & 2559.3 & 2601.2 \\
\hline & PM 2.5t/ha & 1963.8 & 1096.1 & 1961.8 & 1980.3 & 2942.8 & 2975.8 \\
\hline & PM 5.0t/ha & 904.6 & 911.3 & 1585.8 & 1566.7 & 2887.4 & 2880.4 \\
\hline & NPK200 +PM 2.5 & 935.8 & 922.2 & 1924.9 & 1904.6 & 2378.7 & 2360.5 \\
\hline \multirow[t]{6}{*}{$\mathrm{Co}+\mathrm{Me}+\mathrm{Ma}$} & $0 \mathrm{~kg} / \mathrm{ha}$ & 875.6 & 866.5 & 1266.0 & 1301.1 & 1899.0 & 1886.8 \\
\hline & NPK200kg/ha & 1006.6 & 1004.6 & 1816.0 & 1798.8 & 2724.0 & 2628.4 \\
\hline & NPK400kg/ha & 927.9 & 917.4 & 1565.7 & 1499.2 & 2348.5 & 2339.2 \\
\hline & PM 2.5t/ha & 1150.3 & 1210.8 & 1892.6 & 1821.2 & 2839.0 & 2914.5 \\
\hline & PM 5.0t/ha & 1062.8 & 1044.5 & 1500.4 & 1455.6 & 2250.6 & 2250.1 \\
\hline & NPK200 +PM 2.5 & 1047.7 & 1026.6 & 1816.7 & 1801.2 & 2725.1 & 2645.2 \\
\hline LSD (0.05) Crop mixture & & NS & NS & NS & NS & 349.4 & 325.6 \\
\hline LSD (0.05) Fertilizer levels & & 14.7 & 15.1 & NS & NS & 9.24 & 9.54 \\
\hline
\end{tabular}

Co = Cocoyam; $\mathrm{Me}=$ Melon; Ma = Maize; NPK = Nitrogen Phosphorus Potassium; 
Table 3. Effect of intercrop and fertilizer levels on the stem girth of Cocoyam

\begin{tabular}{|c|c|c|c|c|c|c|c|}
\hline \multicolumn{2}{|c|}{ Treatments } & \multicolumn{6}{|c|}{$\begin{array}{c}\text { Months After Planting (cm) } \\
\text { (MAP) }\end{array}$} \\
\hline \multirow[b]{2}{*}{ Crop mixtures } & \multirow[b]{2}{*}{ Fertilizer levels } & \multicolumn{2}{|c|}{2} & \multicolumn{2}{|c|}{4} & \multicolumn{2}{|c|}{6} \\
\hline & & 2018 & 2019 & 2018 & 2019 & 2018 & 2019 \\
\hline \multirow[t]{6}{*}{ Sole cocoyam } & $0 \mathrm{~kg} / \mathrm{ha}$ & 10.4 & 10.2 & 18.2 & 18.1 & 27.3 & 26.8 \\
\hline & NPK $200 \mathrm{~kg} / \mathrm{ha}$ & 12.3 & 12.0 & 21.5 & 21.0 & 32.3 & 31.6 \\
\hline & NPK400kg/ha & 11.1 & 11.3 & 20.2 & 19.8 & 30.3 & 29.9 \\
\hline & PM 2.5t/ha & 14.4 & 14.2 & 22.9 & 22.7 & 34.3 & 34.0 \\
\hline & PM 5.0t/ha & 10.4 & 10.1 & 19.9 & 19.0 & 29.9 & 28.1 \\
\hline & NPK200 +PM 2.5 & 11.6 & 11.8 & 20.1 & 19.9 & 31.0 & 30.1 \\
\hline \multirow[t]{6}{*}{$\mathrm{Co}+\mathrm{Me}$} & 0kg/ha & 7.9 & 7.4 & 18.9 & 18.1 & 28.3 & 27.8 \\
\hline & NPK200kg/ha & 11.3 & 11.0 & 21.2 & 21.0 & 31.8 & 31.4 \\
\hline & NPK $400 \mathrm{~kg} / \mathrm{ha}$ & 9.1 & 9.0 & 20.2 & 20.1 & 30.3 & 30.0 \\
\hline & PM 2.5t/ha & 12.4 & 12.6 & 22.3 & 22.2 & 33.5 & 34.1 \\
\hline & PM 5.0t/ha & 9.7 & 8.9 & 19.6 & 18.9 & 29.5 & 29.7 \\
\hline & NPK200 +PM 2.5 & 10.6 & 9.6 & 20.7 & 20.4 & 31.1 & 31.0 \\
\hline \multirow[t]{6}{*}{$\mathrm{Co}+\mathrm{Ma}$} & $0 \mathrm{~kg} / \mathrm{ha}$ & 8.6 & 8.7 & 18.1 & 18.0 & 27.2 & 27.0 \\
\hline & NPK200kg/ha & 9.9 & 9.7 & 20.5 & 20.2 & 30.8 & 30.6 \\
\hline & NPK400kg/ha & 10.2 & 10.0 & 19.6 & 18.6 & 29.5 & 28.2 \\
\hline & PM 2.5t/ha & 11.4 & 12.0 & 21.3 & 22.1 & 31.9 & 32.3 \\
\hline & PM 5.0t/ha & 9.5 & 9.3 & 19.8 & 18.9 & 29.8 & 28.6 \\
\hline & NPK200 +PM 2.5 & 9.7 & 9.5 & 20.2 & 20.0 & 30.4 & 30.1 \\
\hline \multirow[t]{6}{*}{$\mathrm{Co}+\mathrm{Me}+\mathrm{Ma}$} & $0 \mathrm{~kg} / \mathrm{ha}$ & 8.6 & 8.4 & 18.7 & 18.3 & 28.0 & 28.2 \\
\hline & NPK200kg/ha & 10.7 & 10.6 & 19.9 & 20.0 & 29.9 & 29.7 \\
\hline & NPK400kg/ha & 9.5 & 9.7 & 19.2 & 19.1 & 28.8 & 28.3 \\
\hline & PM 2.5t/ha & 11.0 & 11.2 & 19.7 & 20.1 & 29.6 & 29.8 \\
\hline & PM 5.0t/ha & 8.9 & 8.8 & 19.0 & 18.6 & 28.6 & 27.6 \\
\hline & NPK200 +PM 2.5 & 10.5 & 10.2 & 19.5 & 19.4 & 29.3 & 29.1 \\
\hline LSD(0.05)Crop mixture & & 9.2 & 9.1 & NS & NS & 23.47 & 22.6 \\
\hline LSD(0.05)Fertilizer levels & & 21.6 & 21.5 & 29.73 & NS & 21.84 & 20.7 \\
\hline
\end{tabular}

$\mathrm{Co}=$ Cocoyam $; \mathrm{Me}=$ Melon; $\mathrm{Ma}=$ Maize; $\mathrm{NPK}=$ Nitrogen Phosphorus Potassium;

$\mathrm{PM}=$ Poultry Manure

Table 4. Effect of intercrop and fertilizer levels on the height of Maize

\begin{tabular}{|c|c|c|c|c|c|c|c|}
\hline \multirow{3}{*}{$\begin{array}{l}\text { Treatments } \\
\text { Crop mixtures }\end{array}$} & \multirow[b]{3}{*}{ Fertilizer levels } & \multicolumn{6}{|c|}{ Months After Planting (cm)(MAP) } \\
\hline & & \multicolumn{2}{|r|}{ Mionitu } & \multicolumn{2}{|c|}{4} & \multicolumn{2}{|c|}{6} \\
\hline & & 2018 & 2019 & 2018 & 2019 & 2018 & 2019 \\
\hline \multirow[t]{6}{*}{ Sole maize } & $0 \mathrm{~kg} / \mathrm{ha}$ & 19.9 & 19.2 & 43.3 & 44.4 & 67.5 & 68.4 \\
\hline & NPK200kg/ha & 24.8 & 25.2 & 49.5 & 49.4 & 74.6 & 76.2 \\
\hline & NPK400kg/ha & 29.8 & 30.1 & 59.9 & 59.8 & 92.5 & 93.2 \\
\hline & PM 2.5t/ha & 27.9 & 28.3 & 51.3 & 52.2 & 80.3 & 84.4 \\
\hline & PM 5.0t/ha & 24.1 & $25 `^{\prime} 0$ & 48.7 & 48.8 & 70.6 & 71.4 \\
\hline & NPK200 +PM 2.5 & 26.3 & 27.4 & 52.5 & 50.1 & 76.6 & 80.1 \\
\hline \multirow{6}{*}{$\mathrm{Co}+\mathrm{Ma}$} & $0 \mathrm{~kg} / \mathrm{ha}$ & 19.3 & 19.8 & 40.3 & 40.5 & 85.7 & 84.6 \\
\hline & NPK200kg/ha & 23.7 & 24.4 & 47.4 & 49.8 & 71.0 & 72.2 \\
\hline & NPK $400 \mathrm{~kg} / \mathrm{ha}$ & 22.7 & 23.5 & 44.9 & 45.4 & 69.8 & 68.2 \\
\hline & PM 2.5t/ha & 23.4 & 24.0 & 47.2 & 49.3 & 70.1 & 69.8 \\
\hline & PM 5.0t/ha & 22.5 & 23.1 & 45.1 & 46.1 & 68.5 & 68.1 \\
\hline & NPK200 +PM 2.5 & 26.0 & 27.2 & 51.2 & 52.2 & 76.4 & 77.1 \\
\hline \multirow[t]{6}{*}{$\mathrm{Co}+\mathrm{Ma}+\mathrm{Me}$} & 0kg/ha & 18.1 & 18.5 & 39.0 & 38.8 & 63.4 & 60.4 \\
\hline & NPK200kg/ha & 25.0 & 24.7 & 50.1 & 50.0 & 74.5 & 72.6 \\
\hline & NPK400kg/ha & 24.3 & 24.1 & 48.9 & 48.6 & 72.3 & 70.8 \\
\hline & PM 2.5t/ha & 23.8 & 23.5 & 47.8 & 46.8 & 69.6 & 68.2 \\
\hline & PM 5.0t/ha & 23.6 & 23.1 & 47.6 & 46.2 & 72.2 & 70.6 \\
\hline & NPK200 +PM 2.5 & 25.0 & 24.8 & 50.2 & 50.1 & 74.1 & 72.4 \\
\hline LSD (0.05) Crop mixture & & 51.4 & 43.5 & 107.8 & 104.4 & 103.9 & 106.4 \\
\hline LSD (0.05) Fertilizer levels & & 6.3 & 5.6 & 5.4 & 5.1 & 4.8 & 3.7 \\
\hline
\end{tabular}

$\mathrm{Co}=$ Cocoyam; $\mathrm{Me}=$ Melon; $\mathrm{Ma}=$ Maize; NPK = Nitrogen Phosphorus Potassium;

$\mathrm{PM}=$ Poultry Manure

NPK200kg/ha gave higher values of stem girth with cocoyam + maize + melon at 4, 6 MAP in both years.
The effect of intercrop and fertilizer levels on the height of maize is shown on Table 4. Results showed significant 
Table 5. Effect of intercrop and fertilizer levels on the leaf area of Maize

\begin{tabular}{|c|c|c|c|c|c|c|c|}
\hline \multirow{3}{*}{$\begin{array}{l}\text { Treatments } \\
\text { Crop Mixtures }\end{array}$} & \multirow[b]{3}{*}{ Fertilizer Levels } & \multicolumn{6}{|c|}{ Months After Planting $\left(\mathrm{cm}^{2}\right)$ (MAP) } \\
\hline & & \multicolumn{2}{|c|}{2} & \multicolumn{2}{|l|}{4} & \multicolumn{2}{|l|}{6} \\
\hline & & 2018 & 2019 & 2018 & 2019 & 2018 & 2019 \\
\hline \multirow{6}{*}{ Sole maize } & $0 \mathrm{~kg} / \mathrm{ha}$ & 50.7 & 50.2 & 178.4 & 176.4 & 444.1 & 443.2 \\
\hline & NPK200kg/ha & 52.9 & 51.7 & 213.7 & 214.1 & 455.4 & 454.1 \\
\hline & NPK400kg/ha & 66.1 & 65.8 & 208.6 & 208.1 & 466.6 & 467.2 \\
\hline & PM 2.5t/ha & 47.6 & 46.5 & 194.5 & 193.5 & 424.3 & 425.1 \\
\hline & PM 5.0t/ha & 50.9 & 50.4 & 207.0 & 207.1 & 466.7 & 466.8 \\
\hline & NPK200 +PM 2.5 & 41.2 & 40.6 & 157.9 & 156.8 & 381.8 & 380.7 \\
\hline \multirow[t]{6}{*}{$\mathrm{Co}+\mathrm{Ma}$} & $0 \mathrm{~kg} / \mathrm{ha}$ & 48.6 & 47.8 & 179.6 & 178.4 & 448.8 & 446.8 \\
\hline & NPK200kg/ha & 53.1 & 52.6 & 214.0 & 213.6 & 478.5 & 477.6 \\
\hline & NPK400kg/ha & 46.2 & 46.6 & 185.3 & 186.2 & 417.0 & 416.4 \\
\hline & PM 2.5t/ha & 45.4 & 45.2 & 181.4 & 182.4 & 411.5 & 412.3 \\
\hline & PM 5.0t/ha & 34.3 & 33.6 & 137.1 & 135.2 & 311.9 & 310.6 \\
\hline & NPK200 +PM 2.5 & 38.8 & 38.5 & 154.8 & 155.4 & 335.9 & 334.4 \\
\hline \multirow[t]{6}{*}{$\mathrm{Co}+\mathrm{Ma}+\mathrm{Me}$} & $0 \mathrm{~kg} / \mathrm{ha}$ & 40.7 & 40.2 & 152.7 & 152.2 & 405.4 & 402.4 \\
\hline & NPK $200 \mathrm{~kg} / \mathrm{ha}$ & 44.6 & 43.8 & 179.0 & 178.5 & 408.3 & 408.8 \\
\hline & NPK400kg/ha & 43.6 & 43.1 & 190.3 & 191.1 & 417.4 & 418.4 \\
\hline & PM 2.5t/ha & 39.9 & 38.8 & 155.2 & 155.8 & 368.1 & 367.2 \\
\hline & PM 5.0t/ha & 42.9 & 41.2 & 167.0 & 168.2 & 404.8 & 405.1 \\
\hline & NPK200 +PM 2.5 & 36.6 & 36.4 & 146.5 & 147.1 & 331.7 & 333.2 \\
\hline LSD (0.05)Crop mixture & & NS & NS & NS & NS & NS & NS \\
\hline LSD (0.05) Fertilizer levels & & 3.9 & 2.6 & 3.06 & 3.10 & NS & NS \\
\hline
\end{tabular}

Co = Cocoyam; Me = Melon; Ma = Maize; NPK = Nitrogen Phosphorus Potassium; $\mathrm{PM}=$ Poultry Manure

Table 6. Effect of intercrop and fertilizer levels on the stem girth of Maize

\begin{tabular}{|c|c|c|c|c|c|c|c|}
\hline \multirow{3}{*}{$\begin{array}{l}\text { Treatments } \\
\text { Crop Mixtures }\end{array}$} & \multirow[b]{3}{*}{ Fertilizer Levels } & \multicolumn{6}{|c|}{ Months After Planting (MAP) } \\
\hline & & \multicolumn{2}{|c|}{2} & \multicolumn{2}{|c|}{4} & \multicolumn{2}{|c|}{6} \\
\hline & & 2018 & 2019 & 2018 & 2019 & 2018 & 2019 \\
\hline \multirow{6}{*}{ Sole maize } & $0 \mathrm{~kg} / \mathrm{ha}$ & 2.3 & 2.3 & 4.6 & 4.4 & 7.0 & 6.7 \\
\hline & NPK200kg/ha & 2.6 & 2.7 & 5.3 & 5.2 & 8.0 & 7.9 \\
\hline & NPK400kg/ha & 2.3 & 2.2 & 4.4 & 4.4 & 6.8 & 6.7 \\
\hline & PM 2.5t/ha & 2.8 & 2.9 & 5.7 & 5.6 & 8.4 & 8.2 \\
\hline & PM 5.0t/ha & 2.2 & 2.1 & 4.4 & 4.3 & 6.6 & 6.4 \\
\hline & NPK200 +PM 2.5 & 2.3 & 2.2 & 4.4 & 4.3 & 7.0 & 6.8 \\
\hline \multirow[t]{6}{*}{$\mathrm{Co}+\mathrm{Ma}$} & $0 \mathrm{~kg} / \mathrm{ha}$ & 2.1 & 2.1 & 4.5 & 4.4 & 6.3 & 6.1 \\
\hline & NPK200kg/ha & 2.3 & 2.2 & 4.6 & 4.5 & 7.0 & 6.9 \\
\hline & NPK400kg/ha & 2.1 & 2.1 & 4.8 & 4.7 & 6.6 & 6.4 \\
\hline & PM 2.5t/ha & 2.4 & 2.5 & 4.6 & 4.5 & 7.2 & 7.1 \\
\hline & PM 5.0t/ha & 2.2 & 2.3 & 4.5 & 4.4 & 6.6 & 6.4 \\
\hline & NPK200 +PM 2.5 & 2.3 & 2.2 & 4.7 & 4.6 & 6.7 & 6.5 \\
\hline \multirow{6}{*}{$\mathrm{Co}+\mathrm{Ma}+\mathrm{Me}$} & $0 \mathrm{~kg} / \mathrm{ha}$ & 2.0 & 2.0 & 4.0 & 4.0 & 6.2 & 6.0 \\
\hline & NPK200kg/ha & 2.2 & 2.1 & 4.5 & 4.6 & 7.0 & 6.9 \\
\hline & NPK $400 \mathrm{~kg} / \mathrm{ha}$ & 2.1 & 2.2 & 4.3 & 4.4 & 6.4 & 6.2 \\
\hline & PM 2.5t/ha & 2.2 & 2.3 & 4.4 & 4.5 & 6.8 & 6.5 \\
\hline & PM 5.0t/ha & 2.1 & 2.0 & 4.2 & 4.3 & 6.3 & 6.1 \\
\hline & NPK200 +PM 2.5 & 2.1 & 2.1 & 4.3 & 4.4 & 6.4 & 6.2 \\
\hline LSD (0.05)Crop mixture & & NS & NS & NS & NS & NS & NS \\
\hline LSD (0.05) Fertilizer levels & & NS & NS & NS & NS & NS & NS \\
\hline
\end{tabular}

Co = Cocoyam $;$ Me = Melon; $\mathrm{Ma}=$ Maize $; \mathrm{NPK}=$ Nitrogen Phosphorus Potassium; $\mathrm{PM}=$ Poultry Manure

differences on the intercrop and fertilizer levels at 2,4,6 WAP in both planting seasons. NPK400kg/ha gave higher values of height of maize with sole maize, cocoyam + maize, cocoyam + maize + melon whereas Pm5.0t/ha gave least value with cocoyam + maize and cocoyam + maize + melon at 2, 4, 6 WAP in both seasons.

Table 5 showed the effect of intercrop and fertilizer levels on the leaf area of maize. Results showed significant differences on the fertilizer levels at 2, 4 WAP in both planting seasons. With sole maize, NPK400kg/ha gave higher values of leaf area at 2, 4 WAP whereas Pm5.0t/ha recorded a higher value at 6 WAP in both years. For cocoyam + maize and cocoyam + maize + melon, $\mathrm{NPK} 400 \mathrm{~kg} / \mathrm{ha}$ gave higher values of leaf area of maize at 2 , 
Table 7. Effect of intercrop and fertilizer levels on the vine length of Melon

\begin{tabular}{|c|c|c|c|c|c|c|c|}
\hline \multirow{3}{*}{$\begin{array}{l}\text { Treatments } \\
\text { Crop mixtures }\end{array}$} & \multirow[b]{3}{*}{ Fertilizer levels } & \multicolumn{6}{|c|}{ Months After Planting (cm)(MAP) } \\
\hline & & \multicolumn{2}{|c|}{2} & \multicolumn{2}{|c|}{4} & \multicolumn{2}{|c|}{6} \\
\hline & & 2018 & 2019 & 2018 & 2019 & 2018 & 2019 \\
\hline \multirow{6}{*}{ Sole melon } & $0 \mathrm{~kg} / \mathrm{ha}$ & 89.9 & 89.7 & 133.5 & 132.4 & 201.4 & 201.1 \\
\hline & NPK200kg/ha & 91.3 & 91.4 & 152.5 & 152.2 & 268.5 & 268.1 \\
\hline & NPK400kg/ha & 92.7 & 92.5 & 156.4 & 156.1 & 249.5 & 249.2 \\
\hline & PM 2.5t/ha & 94.4 & 94.7 & 158.5 & 160.8 & 246.4 & 261.1 \\
\hline & PM 5.0t/ha & 92.1 & 91.2 & 154.2 & 155.2 & 259.5 & 258.2 \\
\hline & NPK200 +PM 2.5 & 96.1 & 95.1 & 162.2 & 162.1 & 253.2 & 251.4 \\
\hline \multirow[t]{6}{*}{$\mathrm{Co}+\mathrm{Me}$} & $0 \mathrm{~kg} / \mathrm{ha}$ & 87.7 & 86.6 & 129.4 & 129.1 & 189.7 & 182.4 \\
\hline & NPK200kg/ha & 89.4 & 89.1 & 148.3 & 147.8 & 234.3 & 234.0 \\
\hline & NPK $400 \mathrm{~kg} / \mathrm{ha}$ & 88.9 & 88.4 & 146.5 & 145.8 & 237.4 & 237.1 \\
\hline & PM 2.5t/ha & 87.8 & 88.8 & 144.6 & 144.1 & 239.2 & 244.6 \\
\hline & PM 5.0t/ha & 88.3 & 88.1 & 148.6 & 146.4 & 242.4 & 240.1 \\
\hline & NPK200 + PM 2.5 & 88.1 & 87.4 & 146.3 & 145.5 & 236.3 & 235.3 \\
\hline \multirow[t]{6}{*}{$\mathrm{Co}+\mathrm{Me}+\mathrm{Ma}$} & $0 \mathrm{~kg} / \mathrm{ha}$ & 85.1 & 84.4 & 124.1 & 122.5 & 178.4 & 177.5 \\
\hline & NPK200kg/ha & 86.1 & 86.2 & 142.5 & 142.1 & 230.2 & 239.4 \\
\hline & NPK400kg/ha & 85.5 & 85.1 & 140.6 & 141.3 & 239.3 & 230.2 \\
\hline & PM 2.5t/ha & 87.0 & 87.8 & 139.3 & 140.2 & 231.2 & 231.1 \\
\hline & PM 5.0t/ha & 84.1 & 84.0 & 137.9 & 138.2 & 230.6 & 230.4 \\
\hline & NPK200 +PM 2.5 & 76.5 & 76.2 & 122.9 & 123.0 & 214.5 & 213.6 \\
\hline LSD (0.05)Crop mixture & & 739.6 & 612.4 & 1510.1 & 1306.3 & 5736.8 & 3407.3 \\
\hline LSD (0.05) Fertilizer levels & & 39.4 & 27.5 & 95.1 & 75.3 & 20.4 & 18.4 \\
\hline
\end{tabular}

$\mathrm{Co}=$ Cocoyam $; \mathrm{Me}=$ Melon $; \mathrm{Ma}=$ Maize $; \mathrm{NPK}=$ Nitrogen Phosphorus Potassium;

$\mathrm{PM}=$ Poultry Manure

\section{4, 6 WAP in both seasons.}

The effect of intercrop and fertilizer levels on the stem girth of maize is shown in Table 6. Results showed no significant difference $(\mathrm{P}<0.05)$ on the intercrop and fertilizer levels. However, the fertilizer level of Pm2.5t/ha gave higher values of stem girth with sole crop, cocoyam + maize and cocoyam + maize + melon at 2, 4, 6 WAP in both seasons.

Table 7 showed the effect of intercrop and fertilizer levels on the vine length of melon. The results indicated significant differences $(\mathrm{P}<0.05)$ on the intercrop and fertilizer levels in both planting seasons. For sole cropping system, the mixed fertilizer levels (NPK200 + Pm2.5) gave higher values of vine length at 2, 4 WAP in comparison with the control, whereas NPK200 $\mathrm{kg} / \mathrm{ha}$ recorded a higher value at 6 WAP. The mean values increase at 2, 4, 6 WAP to 92.8, 152.9 and 246.4 respectively for the sole crop. For the intercrop of melon + cocoyam, NPK200kg/ha fertilizer level gave a higher value at $2 \mathrm{WAP}$, whereas Pm5.0t/ha recorded higher values of vine length at 4, 6 WAP respectively. For melon + maize + cocoyam crop mixtures, $\mathrm{Pm} 2.5 \mathrm{t} /$ ha gave a high value of $87.0 \mathrm{~cm}$ at $2 \mathrm{WAP}$. At $4 \mathrm{WAP}, \mathrm{NPK} 200 \mathrm{~kg} / \mathrm{ha}$ recorded $142.5 \mathrm{~cm}$ whereas $N P K 400 \mathrm{~kg} /$ ha gave $239.3 \mathrm{~cm}$ at 6 WAP respectively with melon + maize + cocoyam. The mean values of the vine length increases at 2, 4, 6 WAP in the intercrop adopted within the planting seasons.

Table 8 showed the effect of intercrop and fertilizer levels on the yield and yield components of cocoyam. Results indicated significant differences $(\mathrm{P}<0.05)$ on the corm yield, weight of corms and cormels on both intercrop and fertilizer levels respectively, whereas corm and cormels length, number of cormels showed significant differences
$(\mathrm{P}<0.05)$ on the fertilizer levels. For sole cocoyam, Pm5.0t/ha gave a higher value of corm yield, Weight of corm and number of cormels whereas cormels yield, weight of cormels were favoured with NPK200kg/ha and NPK400kg/ha respectively in both seasons. Fertilizer mixture (NPK200 + Pm2.5) gave higher values of corm and cormels length in both planting seasons. For cocoyam + melon, Pm5.0t/ha gave high values of corm yield, weight of corm and cormels and number of cormels in both seasons. NPK400kg/ha gave high values of cormels yield, length of corm and cormels in both seasons. For cocoyam + maize, NPK400kg/ha gave high values of corm, cormels yield and number of cormels. Pm2.5t/ha gave high values of weight of corm, length of corm and cormels whereas fertilizer mixture (NPK200 + Pm2.5) recorded high weight of cormels in both planting seasons. For cocoyam + maize + melon, higher values of corm yield, weight of corm and cormels were recorded with Pm5.0t/ha. Cormels yield, length of corm and cormels were favoured with $\mathrm{Pm} 2.5 \mathrm{t} / \mathrm{ha}$ whereas number of cormels were high with $N P K 400 \mathrm{~kg} / \mathrm{ha}$ in both seasons.

Table 9 showed the effect of intercrop and fertilizer levels on the yield and yield components of maize. Results indicated significant differences $(\mathrm{P}<0.05)$ on the grain yield of maize on both intercrop and fertilizer levels whereas filling cob percentage showed significant differences $(\mathrm{P}<0.05)$ on the fertilizer levels applied. For sole maize, NPK400kg/ha gave high values of weight of un-dehusk maize and filling cob percentage; Pm2.5t/ha gave higher values of weight of dehusk maize, weight of grain per cob and grain yield; NPK200kg/ha favoured the length of cob in both planting seasons. For maize + cocoyam, NPK200kg/ha 
Table 8. Effect of intercrop and fertilizer levels on the yield and yield components of cocoyam

\begin{tabular}{|c|c|c|c|c|c|c|c|c|c|c|c|c|c|}
\hline \multicolumn{2}{|c|}{ Treatments } & \multicolumn{12}{|c|}{ Yield and Yield Components } \\
\hline \multirow[t]{2}{*}{ Crop mixtures } & \multirow[t]{2}{*}{ Fertilizer levels } & \multicolumn{2}{|c|}{$\begin{array}{c}\text { Corm yield } \\
\text { (t/ha) }\end{array}$} & \multicolumn{2}{|c|}{$\begin{array}{c}\text { Cormels } \\
\text { yield (t/ha) }\end{array}$} & \multicolumn{2}{|c|}{$\begin{array}{l}\text { Wt. of corm } \\
\text { (gms) }\end{array}$} & \multicolumn{2}{|c|}{$\begin{array}{c}\text { Wt. of } \\
\text { Cormels } \\
\text { (kgs) }\end{array}$} & \multicolumn{2}{|c|}{$\begin{array}{c}\text { No. of } \\
\text { cormels }\end{array}$} & \multicolumn{2}{|c|}{$\begin{array}{c}\text { Length of } \\
\text { Cormels }(\mathrm{cm})\end{array}$} \\
\hline & & 2018 & 2019 & 2018 & 2019 & 2018 & 2019 & 2018 & 2019 & 2018 & 2019 & 2018 & 2019 \\
\hline \multirow[t]{6}{*}{ Sole cocoyam } & $0 \mathrm{~kg} / \mathrm{ha}$ & 4.1 & 3.1 & 3.8 & 3.5 & 0.52 & 0.37 & 0.61 & 0.54 & 6.7 & 5.6 & 8.7 & 7.8 \\
\hline & NPK200kg/ha & 5.6 & 5.2 & 5.2 & 4.4 & 0.14 & 0.31 & 0.34 & 0.40 & 8.0 & 7.5 & 7.7 & 6.8 \\
\hline & NPK400kg/ha & 5.9 & 5.3 & 4.1 & 4.0 & 0.48 & 0.46 & 0.83 & 0.48 & 8.0 & 7.3 & 8.2 & 7.6 \\
\hline & PM 2.5t/ha & 5.9 & 5.4 & 3.4 & 3.3 & 0.40 & 0.42 & 0.32 & 0.36 & 4.0 & 4.5 & 6.9 & 6.5 \\
\hline & PM 5.0t/ha & 6.7 & 6.0 & 4.3 & 4.2 & 0.82 & 0.80 & 0.59 & 0.62 & 10.6 & 9.8 & 9.4 & 9.2 \\
\hline & NPK200 +PM 2.5 & 3.9 & 3.3 & 2.9 & 3.0 & 0.63 & 0.56 & 0.55 & 0.48 & 7.6 & 6.7 & 9.6 & 8.1 \\
\hline \multirow[t]{6}{*}{$\mathrm{Co}+\mathrm{Me}$} & $0 \mathrm{~kg} / \mathrm{ha}$ & 4.8 & 3.7 & 2.8 & 2.4 & 0.38 & 0.41 & 0.47 & 0.45 & 7.0 & 6.5 & 7.1 & 6.4 \\
\hline & NPK200kg/ha & 5.9 & 5.4 & 4.3 & 4.5 & 0.33 & 0.38 & 0.46 & 0.54 & 7.0 & 7.8 & 6.8 & 7.6 \\
\hline & NPK400kg/ha & 6.2 & 5.8 & 5.5 & 5.0 & 0.44 & 0.50 & 0.42 & 0.48 & 6.0 & 6.7 & 10.9 & 9.7 \\
\hline & PM 2.5t/ha & 6.5 & 6.1 & 2.7 & 2.5 & 0.67 & 0.70 & 0.45 & 0.51 & 8.0 & 7.8 & 8.6 & 8.4 \\
\hline & PM 5.0t/ha & 7.8 & 8.2 & 3.0 & 2.9 & 0.80 & 0.84 & 0.67 & 0.70 & 9.0 & 8.0 & 8.6 & 8.3 \\
\hline & NPK200 +PM 2.5 & 4.9 & 4.4 & 3.2 & 3.5 & 0.45 & 0.51 & 0.34 & 0.40 & 6.0 & 6.8 & 6.6 & 6.8 \\
\hline \multirow[t]{6}{*}{$\mathrm{Co}+\mathrm{Ma}$} & $0 \mathrm{~kg} / \mathrm{ha}$ & 3.5 & 3.2 & 2.5 & 2.8 & 0.26 & 0.28 & 0.31 & 0.30 & 6.0 & 5.6 & 6.9 & 6.4 \\
\hline & NPK200kg/ha & 4.2 & 4.0 & 3.5 & 4.0 & 0.31 & 0.42 & 0.30 & 0.31 & 6.0 & 7.2 & 7.1 & 6.6 \\
\hline & NPK400kg/ha & 6.1 & 5.5 & 3.5 & 3.6 & 0.30 & 0.40 & 0.37 & 0.37 & 7.0 & 7.5 & 7.5 & 7.0 \\
\hline & PM 2.5t/ha & 5.8 & 6.2 & 3.3 & 3.2 & 0.64 & 0.61 & 0.35 & 0.39 & 6.0 & 7.2 & 8.4 & 8.1 \\
\hline & PM 5.0t/ha & 5.9 & 5.7 & 2.7 & 3.0 & 0.36 & 0.47 & 0.32 & 0.34 & 7.0 & 8.0 & 7.5 & 7.0 \\
\hline & NPK200 +PM 2.5 & 4.1 & 3.8 & 2.6 & 2.8 & 0.61 & 0.56 & 0.39 & 0.38 & 4.0 & 4.6 & 6.9 & 6.2 \\
\hline \multirow[t]{6}{*}{$\mathrm{Co}+\mathrm{Me}+\mathrm{Ma}$} & $0 \mathrm{~kg} / \mathrm{ha}$ & 1.9 & 2.3 & 1.3 & 2.3 & 0.23 & 0.21 & 0.31 & 0.30 & 5.0 & 4.2 & 6.4 & 5.4 \\
\hline & NPK200kg/ha & 3.8 & 4.0 & 2.2 & 2.7 & 0.40 & 0.48 & 0.41 & 0.40 & 6.0 & 5.4 & 6.6 & 7.0 \\
\hline & NPK400kg/ha & 4.5 & 4.9 & 3.0 & 4.0 & 0.36 & 0.45 & 0.33 & 0.37 & 8.0 & 7.2 & 7.8 & 8.0 \\
\hline & PM 2.5t/ha & 5.0 & 5.6 & 5.5 & 6.0 & 0.54 & 0.61 & 0.30 & 0.35 & 6.0 & 5.4 & 7.9 & 8.2 \\
\hline & PM 5.0t/ha & 5.5 & 6.1 & 3.3 & 4.1 & 0.60 & 0.68 & 0.44 & 0.48 & 7.0 & 6.2 & 7.2 & 7.8 \\
\hline & NPK200 +PM 2.5 & 4.2 & 4.4 & 2.5 & 3.0 & 0.44 & 0.54 & 0.42 & 0.41 & 6.0 & 5.6 & 6.2 & 5.4 \\
\hline LSD (0.05) Crop mixture & & \multicolumn{2}{|c|}{51.0} & NS & 6.3 & 7.0 & 5.8 & 7.2 & 8.4 & NS & 4.2 & NS & 3.4 \\
\hline LSD (0.05) Fertilizer levels & & \multicolumn{2}{|c|}{3.3} & NS & NS & 3.8 & 3.6 & 3.0 & 2.6 & 4.1 & 3.5 & 4.2 & 5.1 \\
\hline
\end{tabular}

$\mathrm{Co}=$ Cocoyam $; \mathrm{Me}=$ Melon; $\mathrm{Ma}=$ Maize $; \mathrm{NPK}=$ Nitrogen Phosphorus Potassium;

$\mathrm{PM}=$ Poultry Manure

Table 9. Effect of intercrop and fertilizer levels on the yield and yield components of maize

\begin{tabular}{|c|c|c|c|c|c|c|c|c|c|c|c|c|c|}
\hline \multirow{3}{*}{$\begin{array}{l}\text { Treatments } \\
\text { Crop mixtures }\end{array}$} & \multicolumn{11}{|c|}{ Yield and Yield Components } & \multirow{2}{*}{\multicolumn{2}{|c|}{$\begin{array}{c}\text { Grain yield } \\
\text { (t/ha) }\end{array}$}} \\
\hline & \multirow[t]{2}{*}{ Fertilizer levels } & \multicolumn{2}{|c|}{$\begin{array}{l}\text { Wt. of un- } \\
\text { dehusked } \\
\text { (kg) }\end{array}$} & \multicolumn{2}{|c|}{$\begin{array}{c}\begin{array}{c}\text { Wt. of } \\
\text { dehusked } \\
\text { (kg) }\end{array} \\
\end{array}$} & \multicolumn{2}{|c|}{$\begin{array}{l}\text { Filling cob } \\
\text { percentage }\end{array}$} & \multicolumn{2}{|c|}{$\begin{array}{l}\text { Length of } \\
\operatorname{cob}(\mathrm{cm})\end{array}$} & \multicolumn{2}{|c|}{$\begin{array}{c}\text { Wt. of } \\
\text { grains/cob } \\
\text { (kg) }\end{array}$} & & \\
\hline & & 2018 & 2019 & 2018 & 2019 & 2018 & 2019 & 2018 & 2019 & 2018 & 2019 & 201 & 2019 \\
\hline \multirow[t]{6}{*}{ Sole maize } & $0 \mathrm{~kg} / \mathrm{ha}$ & 0.23 & 0.21 & 0.19 & 0.17 & 70.7 & 63.6 & 17.8 & 15.9 & 0.12 & 0.12 & 4.5 & 4.4 \\
\hline & NPK200kg/ha & 0.24 & 0.23 & 0.19 & 0.18 & 80.7 & 74.6 & 18.9 & 17.6 & 0.15 & 0.16 & 5.2 & 5.1 \\
\hline & NPK400kg/ha & 0.26 & 0.24 & 0.19 & 0.19 & 82.3 & 81.4 & 18.1 & 17.0 & 0.13 & 0.14 & 5.4 & 5.3 \\
\hline & PM 2.5t/ha & 0.26 & 0.26 & 0.21 & 0.22 & 80.0 & 74.3 & 18.4 & 17.2 & 0.15 & 0.16 & 5.6 & 5.4 \\
\hline & PM 5.0t/ha & 0.24 & 0.24 & 0.18 & 0.18 & 81.1 & 75.4 & 16.4 & 15.4 & 0.12 & 0.13 & 5.3 & 5.2 \\
\hline & NPK200 +PM 2.5 & 0.26 & 0.24 & 0.17 & 0.17 & 82.3 & 78.5 & 18.0 & 16.8 & 0.13 & 0.14 & 5.0 & 4.8 \\
\hline \multirow[t]{6}{*}{$\mathrm{Co}+\mathrm{Ma}$} & $0 \mathrm{~kg} / \mathrm{ha}$ & 0.26 & 0.22 & 0.20 & 0.18 & 67.0 & 70.2 & 18.1 & 15.4 & 0.12 & 0.13 & 5.1 & 4.4 \\
\hline & NPK200kg/ha & 0.28 & 0.26 & 0.21 & 0.22 & 81.4 & 80.6 & 18.4 & 18.2 & 0.16 & 0.16 & 5.2 & 5.3 \\
\hline & NPK400kg/ha & 0.25 & 0.24 & 0.20 & 0.20 & 80.5 & 76.8 & 17.6 & 17.9 & 0.13 & 0.14 & 4.2 & 4.1 \\
\hline & PM 2.5t/ha & 0.24 & 0.26 & 0.20 & 0.21 & 80.0 & 74.6 & 17.0 & 16.3 & 0.14 & 0.15 & 5.4 & 5.5 \\
\hline & PM 5.0t/ha & 0.24 & 0.22 & 0.19 & 0.19 & 81.7 & 82.0 & 17.2 & 16.6 & 0.14 & 0.15 & 4.9 & 4.8 \\
\hline & NPK200 +PM 2.5 & 0.22 & 0.24 & 0.18 & 0.18 & 81.2 & 80.2 & 16.9 & 15.8 & 0.12 & 0.13 & 4.9 & 4.8 \\
\hline \multirow[t]{6}{*}{$\mathrm{Co}+\mathrm{Ma}+\mathrm{Me}$} & 0kg/ha & 0.22 & 0.22 & 0.18 & 0.17 & 70.6 & 68.5 & 17.0 & 15.8 & 0.12 & 0.11 & 4.9 & 4.8 \\
\hline & NPK $200 \mathrm{~kg} / \mathrm{ha}$ & 0.23 & 0.24 & 0.19 & 0.18 & 81.4 & 82.1 & 17.6 & 18.4 & 0.13 & 0.14 & 4.8 & 4.7 \\
\hline & NPK400kg/ha & 0.23 & 0.24 & 0.20 & 0.22 & 79.8 & 71.2 & 17.6 & 18.1 & 0.13 & 0.14 & 4.7 & 4.6 \\
\hline & $\mathrm{PM} \mathrm{2.5t/ha}$ & 0.23 & 0.26 & 0.19 & 0.19 & 80.0 & 80.4 & 16.7 & 16.0 & 0.15 & 0.16 & 5.4 & 5.5 \\
\hline & PM 5.0t/ha & 0.23 & 0.23 & 0.17 & 0.17 & 80.6 & 81.1 & 16.8 & 16.2 & 0.11 & 0.12 & 4.6 & 4.4 \\
\hline & NPK200 +PM 2.5 & 0.23 & 0.22 & 0.17 & 0.16 & 79.1 & 70.6 & 17.1 & 16.8 & 0.11 & 0.11 & 4.7 & 4.6 \\
\hline LSD (0.05)Crop mixture & & NS & NS & NS & NS & NS & NS & NS & NS & NS & NS & 7.8 & 6.2 \\
\hline LSD(0.05)Fertilizer levels & & NS & NS & NS & NS & 9.1 & 7.4 & NS & NS & NS & NS & 5.6 & 3.8 \\
\hline
\end{tabular}

Co $=$ Cocoyam $; \mathrm{Me}=$ Melon; $\mathrm{Ma}=$ Maize; $\mathrm{NPK}=$ Nitrogen Phosphorus Potassium;

$\mathrm{PM}=$ Poultry Manure 
Table 10. Effect of intercrop and fertilizer levels on the yield and yield components of melon

\begin{tabular}{|c|c|c|c|c|c|c|c|c|c|c|c|}
\hline \multirow{3}{*}{$\begin{array}{l}\text { Treatments } \\
\text { Crop mixtures }\end{array}$} & \multicolumn{9}{|c|}{ Yield and Yield Components } & & \\
\hline & \multirow[t]{2}{*}{ Fertilizer levels } & \multicolumn{2}{|c|}{$\begin{array}{l}\text { Length of pod } \\
\text { (cm) }\end{array}$} & \multicolumn{2}{|c|}{$\begin{array}{l}\text { Circumference } \\
\text { of pod (cm) }\end{array}$} & \multicolumn{2}{|c|}{$\begin{array}{c}\text { Mean No. of } \\
\text { pod }\end{array}$} & \multicolumn{2}{|c|}{$\begin{array}{c}\text { Melon seed yield } \\
\text { (t/ha) }\end{array}$} & \multicolumn{2}{|c|}{$\begin{array}{c}\text { Total wt. of } \\
\text { pods/ha (t/ha) }\end{array}$} \\
\hline & & 2018 & 2019 & 2018 & 2019 & 2018 & 2019 & 2018 & 2019 & 2018 & 2019 \\
\hline \multirow[t]{6}{*}{ Sole melon } & $0 \mathrm{~kg} / \mathrm{ha}$ & 10.7 & 10.4 & 11.3 & 11.1 & 1.8 & 2.0 & 0.34 & 0.32 & 8.4 & 7.8 \\
\hline & NPK200kg/ha & 11.1 & 11.4 & 12.5 & 12.4 & 3.2 & 3.3 & 0.40 & 0.42 & 10.2 & 9.5 \\
\hline & NPK400kg/ha & 10.9 & 11.0 & 12.6 & 12.5 & 2.3 & 2.5 & 0.37 & 0.37 & 8.3 & 8.4 \\
\hline & PM 2.5t/ha & 11.6 & 11.7 & 12.7 & 12.6 & 2.8 & 2.9 & 0.42 & 0.44 & 8.8 & 8.9 \\
\hline & PM 5.0t/ha & 11.9 & 12.0 & 12.8 & 12.7 & 2.7 & 2.6 & 0.27 & 0.29 & 8.7 & 8.5 \\
\hline & NPK200 +PM 2.5 & 10.8 & 10.6 & 12.3 & 12.1 & 2.0 & 2.2 & 0.37 & 0.34 & 10.3 & 9.8 \\
\hline \multirow[t]{6}{*}{$\mathrm{Co}+\mathrm{Me}$} & 0kg/ha & 10.3 & 10.4 & 11.8 & 11.5 & 1.5 & 1.7 & 0.30 & 0.33 & 7.1 & 8.0 \\
\hline & NPK200kg/ha & 11.5 & 11.2 & 12.2 & 12.4 & 2.5 & 2.6 & 0.38 & 0.40 & 7.2 & 8.2 \\
\hline & NPK400kg/ha & 10.2 & 10.0 & 11.9 & 12.0 & 2.1 & 2.0 & 0.33 & 0.36 & 3.9 & 4.2 \\
\hline & PM 2.5t/ha & 11.5 & 11.2 & 12.5 & 12.7 & 2.2 & 2.3 & 0.32 & 0.35 & 6.8 & 7.1 \\
\hline & PM 5.0t/ha & 11.6 & 11.5 & 12.5 & 12.7 & 2.4 & 2.5 & 0.25 & 0.30 & 10.4 & 10.1 \\
\hline & NPK200 +PM 2.5 & 11.6 & 11.5 & 12.1 & 12.2 & 2.3 & 2.4 & 0.38 & 0.39 & 9.2 & 9.9 \\
\hline \multirow[t]{6}{*}{$\mathrm{Co}+\mathrm{Me}+\mathrm{Ma}$} & $0 \mathrm{~kg} / \mathrm{ha}$ & 10.9 & 11.1 & 10.9 & 10.6 & 1.2 & 1.1 & 0.32 & 0.30 & 8.9 & 6.7 \\
\hline & NPK200kg/ha & 11.2 & 11.4 & 11.2 & 11.4 & 1.8 & 2.0 & 0.27 & 0.26 & 3.0 & 3.6 \\
\hline & NPK400kg/ha & 10.3 & 10.5 & 12.3 & 12.6 & 1.5 & 1.7 & 0.28 & 0.27 & 6.8 & 6.3 \\
\hline & PM 2.5t/ha & 10.6 & 11.0 & 12.0 & 12.1 & 1.4 & 1.5 & 0.27 & 0.26 & 5.4 & 5.8 \\
\hline & PM 5.0t/ha & 12.0 & 11.8 & 12.3 & 12.6 & 1.3 & 1.4 & 0.30 & 0.28 & 6.5 & 6.1 \\
\hline & NPK200 +PM 2.5 & 10.4 & 10.2 & 11.6 & 11.9 & 1.4 & 1.5 & 0.32 & 0.31 & 3.9 & 4.0 \\
\hline LSD (0.05)Crop mixture & & NS & NS & 18.9 & 19.3 & 18.7 & 17.8 & NS & NS & 29.0 & 26.5 \\
\hline LSD (0.05) Fertilizer levels & & 6.4 & 7.1 & NS & NS & 7.1 & 6.4 & NS & NS & 3.7 & 2.8 \\
\hline
\end{tabular}

Co = Cocoyam; $\mathrm{Me}=$ Melon; Ma = Maize; NPK = Nitrogen Phosphorus Potassium;

$\mathrm{PM}=$ Poultry Manure

recorded high values of weight of undehusk and dehusked maize, length of cob and weight of grains per cob. Pm5.0t/ha gave high values of filling cob percentage whereas Pm2.5t/ha recorded high value of grain yield in both planting seasons. For maize + melon + cocoyam, NPK200kg/ha recorded high values of weight of undehusked, filling cob percentage, length of cob. NPK400kg/ha gave high values of weight of dehusked whereas Pm2.5t/ha recorded high values of weight of grains per cob and grain yield in both seasons.

Table 10 showed the effect of intercrop and fertilizer levels on the yield and yield components of melon. Results indicated significant differences $(\mathrm{P}<0.05)$ on the mean number of pods and total weight of the pods on both crop mixtures and fertilizer levels whereas length of pods and circumference of pods showed significant differences $(\mathrm{P}<0.05)$ on the fertilizer levels and crop mixtures respectively. For sole melon, NPK200kg/ha gave high value of mean number of pods, Pm2.5t/ha recorded high value of melon seed yield, Pm5.0t/ha gave high value of length and circumference of pods and fertilizer mixture (NPK200 + Pm2.5) recorded higher total weight of pods in both planting seasons. For melon + cocoyam, NPK $200 \mathrm{~kg} / \mathrm{ha}$ recorded high values of mean number of pods and melon seed yield, Pm5.0t/ha gave higher values of length of pods, circumference of pods and total weight of pods whereas fertilizer mixture (NPK200 + Pm2.5) recorded high melon seeds yield in the two planting seasons. For melon + maize + cocoyam, NPK200kg/ha gave high values of mean number of pods, NPK $400 \mathrm{~kg} / \mathrm{ha}$ recorded high values of circumference of pods and total weight of pods, Pm5.0t/ha gave higher length of pods whereas melon seeds yields were high with fertilizer mixture (NPK200 + Pm2.5) in both seasons.

\section{DISCUSSION}

The use of fertilizer will increase the yield of crops thereby making it possible to obtain maximum yields and this will lead to economic benefit for farmers. From the result, higher values of cocoyam height and maize height were recorded with NPK200kg/ha and NPK400kg/ha respectively in both sole and crop mixtures. This finding however agrees with Azza et al. (2006) that NPK fertilizers can stimulate crop growth and improve its mineral uptake. This was further collaborated by the findings of Smil (2000) who opined that the use of inorganic fertilizer improves crop yields by way of increasing soil fertility and nutrient availability. This explanation holds for the increase in leaf area, stem girth and the number of leaves of cocoyam, maize and melon plant. The significant increase in plant height observed with increase NPK fertilizer when compared with the control could be attributed further to the nitrogen content of the applied fertilizer. The presence of phosphorus in the fertilizer increases the absorption of nitrogen according to Badran and Safwat (2004), Majeed and Ali (2011) which promotes vegetative productions.

The difference in plant heights and stem girths probably reflects the difference in the nature of competition between the sole cropping and the crop mixtures in the systems. It also appeared that the important factors of competition 
were probably light and nutrients.

Intercropping cocoyam with maize and melon tends to promote vegetative phase of cocoyam and maize. The reduction in the leaf area index of the intercrop systems implies that the corm and cormels yields were positively associated with the leaf size both cropping systems and this applies to maize. These agrees with the findings of Igbokwe (1983). Tayo (1982) also noted that the decrease in leaf area index with an advance in the age of cocoyam was due to senescence of old leaves and outgrowth of small ones.

The corm yield of cocoyam was higher with high organic manure (PM 5.0t/ha) when compared with the control. The lower rate of organic manure with the attendant lower corm yield can be attributed to the relative competition among the crops. However, cocoyam with melon crop mixtures enhanced cocoyam yield relative to sole cocoyam. This could be attributable to the short life span of melon and subsequent released of residual nitrogen from the leaves to the soil (Mba, 1983). This further support the findings of Akinyemi and Tijani-Eniola (1997) who opined the subsequent decay of melon foliage provides additional nutrients and improved soil conditions for cocoyam rapid growth and production. Murwira (1993) and Ayoola (2006) had reported improved nutrient use efficiency with organic fertilizer. Cocoyam being a root crop has a high requirement for potassium as yam and cassava (Obiebesan et al., 1976; Kay, 1987; Onwueme and Sinha, 1991). The potassium content of organic manure is usually high though dependent on the type, makes it suitable for root crops. The corm, corm weight, number of cormels and total yield per hectare were optimised with higher rates of organic manure. This result confirms the findings of Ohiri et al.(1988) that cocoyam requires high poultry manure and higher rates of potassium depending on soil type. This assertion agrees with Adediran and Banjoko (2003) who explained that the use of organic fertilizers on crops increased yield.

In maize, the weight of dehusked, dehusked, cob filling percentage and length of cob and grain yield showed variations from sole to intercrop. The result showed higher yield components of maize under sole cropping compared to the crop mixtures. This indicates that crops in sole plots suffer less from competition. The consequence of this findings is that the nutrients requirement of maize in the crop mixtures were higher than the nutrients need of the crop when planted as sole. Baker (1979), Mba et al. (2007) and Ali et al. (2015) further opined that the nutrient demand of the component crops was always higher than for the sole crops. The highest values of the parameters were observed with the application of inorganic fertilizers although organic fertilizers and their combinations showed variations in some specific parameters. Mokwunye (1980) reported when organic manure is applied together with mineral fertilizers, the later aids the decomposition of the former. Other researchers (Lombin and abdullahi, 1977; Lombin et al., 1994; Ayoola and Adeniyan, 2006) also observed that the use of poultry manure increased the efficiency of inorganic fertilizer probably by serving as a liming materials and providing secondary micronutrients not present in the organic fertilizers.

\section{Conclusion and recommendations}

The findings of this study generates the following conclusion.

Organic fertilizer mostly at PM 5.0t/ha promoted the yield and yield components of cocoyam (corms yields, cormels yields, weight of corm, weight of cormels, number of cormels and length of cormels) in both sole cocoyam and cocoyam crop mixtures.

In sole maize and maize crop mixtures, organic manure PM2.5t/ha gave higher yields of weight of undehusked, weight of dehusked, weight of grain and grain yield per hectare whereas inorganic fertilizers (NPK200kg/ha and NPK400kg/ha) favoured the filling cob percentage and length of cob.

Generally, a combination of both organic and inorganic fertilizer has proven effective for maize yield and yield components.

\section{Conflict of interests}

The authors declare that they have no conflicting interests.

\section{REFERENCES}

Adediran JA, Banjoko VA (2003). Comparative effectiveness of some compost fertilizer formulations for maize in Nigeria. Nigerian J. Soil Science. 13: $42-48$.

Akinyemi SS, Tijani- Eniola H (1997). Effect of melon population density intercropped with Plantain on weed control and intercrop productivity. Nigerian J. weed Sci. 10:27 -34.

Ayoola OT, Adeniyan ON (2006). Influence of poultry on yield and yield components of crops under different cropping systems in South West Nigeria. African J. Biotechnol, 5: 1386 - 1392.

Azza AMM, Yassen AA, Sahar MZ (2006). Impact of boron fertilizer on growth under water regime. World J. Agric. Sci.2 (4): $412-420$.

Badran FS, Safwat MS (2004). Response of fennal plants to organic manure and bio-fertilizers in replacement of chemical fertilization in Egypt. J. Agric. Res. 82(8): 247 256.

Barker EFI (1979). Research on mixed cropping with cereals in Nigeria farming systems - a system for improvement. In: International workshop on farming systems. Proceedings ICRISAT Hyderabad, November, $18^{\text {th }}-21^{\text {st }}$, pp $287-301$

Doku EV (1988). West African tropical root crop farming systems. Proceedings of the $3^{\text {rd }}$ West African root crops workshop held in Accra, Ghana, July $22-26^{\text {th }} 1985$. R. C. Therberge (ed) IITA, Ibadan, Nigeria.

Economic Commission of Africa (2011). State of the Environment in Africa. Economic Commission of Africa, 
Addis Ababa, Ethiopia. Eca/fssdd/01/06. http://www.uncea.org/water/state.environ.afri.

Igbokwe MC (1983). Growth and development of colocasia and xanthosoma spp under upland condition. Proceedings of the $2^{\text {nd }}$ symposium. ISTEC.AB,Duala, Cameroun: 14 19th August 1983. $182-184$.

Kay ED (1987). Root crops: Crop and product digest No.2, pp $227-228$.

Lombin HC, Abdullahi KA (1977). In: Fertilizer use and management practices for crops in Nigeria produced by Federal Fertilizer Department, Abuja, 2002

Lombin LG, Adepetu JA, Ayotade KA (1994). In: Organic fertilizer in the Nigerian Agriculture: Present and Future, FPDD Abuja, Nigeria, pp 146 - 162.

Majeed KA, Ali SA (2011). Effect of foliar application of NPK 20:10:10 on some growth characters of food cultivars of Roselle (Hibiscus sabdariffa L). American J. Plant Physiol. 6: 220 - 227

Mba CC (1983). Egusi plant - Colocynthis citrullus and soil properties, In: Obiefuna, J. C. (1989). Biological weed control in plantains (musa AAB) with egusi melon (Colocynthis citrullus). Biological Agriculture and Horticulture 6: 221 -227.

Mokwunye U (1980). In: Organic cycling in African soils. Bulletin - FAO, Rome,No. 43: 192 - 200

Murwira HK, Kirchmenn H (1993). Soil organic matter dynamics and sustainability of tropical Agriculture. Pp189 - 198.

Obigbesan GO (1976). Effect of potassium on tuber yield and nutrient uptake yam varieties. Proceedings of the $4^{\text {th }}$ symposium of the International Society for Root Crops. IDRCCIAT, Columbia. Ed. Cook, Macintyre and Graham. $104-107$.

Ofori F Stern WR (1987). Cereal - legume intercropping systems. Advances in Agronomy 14: 41 - 89.

Ohiri AC, Ogbonaya JC, Enyineya AM, Ojinaka TL, Chukwu GO (1988). Annual Report, NRCRI, Umudike, pp 75 - 77.

Oluma G (2010). Sustainable horticultural production through intercropping: A review. Agricultural Biology I.N.S. 1098 - 1103.

Onwueme IC, Sinha TD (1991). Field crop production in Tropical Africa. pp 276 - 287.

Smil V (2000). Phosphorus in the environment. National Flaws and Human Inferences.Annual Review of Energy and Environment 28: $609-620$.

Tayo OT (1982). Growth, yield and development of pigeon pea (Cajanus cajan) in lowland tropics. In: Effect of early loss of apical dominances. J. Agric. Sci. 98: $79-84$.

Udounang PI (2007). Response of cocoyam-based intercropping systems to organic and inorganic fertilizers in Obio Akpa, South Eastern Nigeria. M.Sc. Thesis, University of Uyo, Uyo, Akwa Ibom State.

Udounang PI (2015). Response of cocoyam-based intercropping systems to tillage practices and fertilization in Uyo, South Eastern Nigeria. Ph.D Dissertation, University of Uyo, Uyo, Akwa Ibom State.

Wong P, Salmah YHM, Cheman YB (2003). Physico-chemical characteristics of Roselle (Hibiscus sabdariffa L). Nutritional Food Sci. 32: 68 - 73. 